\title{
The nature of comet-asteroid transition object (3200) Phaethon
}

\author{
J. Licandro ${ }^{1,2}$, H. Campins ${ }^{3}$, T. Mothé-Diniz ${ }^{4}$, N. Pinilla-Alonso ${ }^{5}$, and J. de León ${ }^{2}$ \\ 1 Isaac Newton Group, PO Box 321, 38700 Santa Cruz de La Palma, Tenerife, Spain \\ e-mail: licandro@ing.iac.es \\ 2 Instituto de Astrofísica de Canarias, c/vía Láctea s/n, 38205 La Laguna, Tenerife, Spain \\ 3 University of Central Florida, Orlando, FL 32765, USA \\ 4 Observatório Nacional/MCT, Rua Gal. José Cristino 77, Rio de Janeiro 20921-400, RJ, Brazil \\ 5 Centro Galileo Galilei \& Telescopio Nazionale Galileo, PO Box 565, 38700 S/C de La Palma, Tenerife, Spain
}

Received 15 June 2006 / Accepted 5 September 2006

\section{ABSTRACT}

\begin{abstract}
Context. The study of asteroids that present sporadic cometary activity is of fundamental importance to address several astronomical problems including the end states of comet nuclei, the abundance of water in main belt asteroids, and its role as a possible source of terrestial water.

Aims. We studied the composition of the surface of asteroid (3200) Phaethon, a paradigmatic case of asteroid-comet transition object, in order to determine its cometary or asteroidal nature.

Methods. We report visible and near infrared spectra covering the 0.35-2.4 $\mu \mathrm{m}$ spectral range, obtained with the $4.2 \mathrm{~m}$ William Herschel Telescope, the $2.5 \mathrm{~m}$ Nordic Optical Telescope, and the Italian $3.58 \mathrm{~m}$ Telescopio Nazionale Galileo at "El Roque de los Muchachos" Observatory (La Palma, Spain). Our spectrum is compared with those of meteorite samples and man-made mineral mixtures to determine possible components, modeled using multiple scattering formulations, and also compared with the spectra of comet nuclei and other comet-asteroid transitional objects.

Results. Phaethon's spectrum does not show any sharp structure and has a negative slope at wavelengths $>0.43 \mu \mathrm{m}$, consistent with B-type asteroids. Below $0.43 \mu \mathrm{m}$ the reflectance decreases. The spectral shape is similar to that of aqueously altered CI/CM meteorites and of hydrated minerals. A surface composition with hydrated silicates is also suggested by the models. A possible spectral variability in the UV is suggested by the avaliable spectra, and is compatible with a slightly different abundance of hydrated silicates. Finally, Phaethon's spectrum shows important differences with the few comet nuclei properly observed at these wavelengths and is similar to the spectra of other peculiar comet-asteroid transition objects.

Conclusions. The spectral properties and dynamical properties of (3200) Phaethon support an asteroidal nature rather than a cometary one. Phaethon is more likely an "activated" asteroid, similar to the population of activated asteroids in the Main Belt Comets, than an extinct comet.
\end{abstract}

Key words. minor planets, asteroids - comets: general

\section{Introduction}

The Apollo asteroid (3200) Phaethon (also known as 1983 TB), was discovered by the IRAS satellite in October 1983 (Green 1983) and has a very unusual orbit, with perihelion at $0.14 \mathrm{AU}$, a semimajor axis of $1.27 \mathrm{AU}$, and an eccentricity of 0.89 . It was the first asteroid to be associated with a meteor shower. Whipple (1983) identified Phaethon as the likely parent of the Geminid meteor stream because its orbital elements are virtually coincident with the mean orbital elements of 19 Geminid meteors with photographically established orbits. Typically, meteor streams are associated with the orbits of active comets, e.g., 1P/Halley (Orionids), 2P/Encke (Taurids), 55P/Tempel-Tuttle (Leonids), and 109P/Swift-tuttle (Perseids); however, Phaethon has not displayed cometary activity (e.g., Cochran \& Barker 1984; Davies et al. 1984; Chamberlin et al. 1996; Hsieh \& Jewitt 2005). Although it is possible that the Geminids formed following a collision between Phaethon and an asteroid, several models favor the ejection of meteoroids under circumstances (location, speed, and directions of ejection) that are possible or even expected during cometary activity (Gustafson 1989; Williams \& Wu 1993). Also, Hunt et al. (1985) proposed an asteroidal collisional model for the shower, but it appears to be unable to reproduce the observed distribution of aphelia. In addition, there are several recent developments relevant to the case of 3200 Phaethon. Two other Near-Earth Asteroids (NEAs), 2001 YB5 and 2005 UD, have been associated with meteor showers (Meng et al. 2004; and Ohtsuka et al. 2006, respectively). Another relevant NEA is 4015 Wilson-Harrington (W-H, also know as comet 107P), which was first dicovered as a comet and re-discovered as an asteroid (Bowell et al. 1992). W-H is a potential meteorite producing object (Campins \& Swindle 1998). A separate set of possibly related objects are those labeled "main belt comets" (MBCs) by Hsieh \& Jewitt (2006), which include 133P/Elst-Pizarro. Two of the three MBCs known to date are within the Themis family of asteroids and the third one is almost within that family.

From a dynamical point of view, Phaethon appears to be of asteroidal origin. According to a model by Bottke et al. (2002), Phaethon has a $0 \%$ chance of coming from the Jupiter family (JF) of comets or from the Oort Cloud, and it more likely originated in the $v 6$ resonance of the main belt of asteroids.

In this paper, we present new visible and near-ir spectra of (3200) Phaethon, covering the $0.35-2.4 \mu \mathrm{m}$ spectral region, and we use these spectra to derive mineralogical information about its surface. 
Table 1. Log of observations: $\alpha$ is the phase angle; wl is the wavelength range of the spectrum; \#spec is the number of spectra obtained; Exp. time is the exposure time of each individual spectrum.

\begin{tabular}{llccccc}
\hline \hline Tel. & $\begin{array}{l}\text { Date } \\
(\mathrm{UT})\end{array}$ & $\begin{array}{c}\alpha \\
(\mathrm{deg})\end{array}$ & $\begin{array}{c}\text { wl } \\
(\mu \mathrm{m})\end{array}$ & \#spec & $\begin{array}{c}\text { Exp. time } \\
(\mathrm{s})\end{array}$ & Airmass \\
\hline WHT & $2003 / 11 / 14.844$ & 56.9 & $0.35-0.95$ & 3 & $300 \mathrm{~s}$ & 1.20 \\
TNG & $2004 / 12 / 11.917$ & 18.2 & $0.8-2.4$ & 8 & $60 \mathrm{~s}$ & 1.02 \\
NOT & $2004 / 12 / 20.97$ & 30.9 & $0.35-0.6$ & 1 & $900 \mathrm{~s}$ & 1.09 \\
NOT & $2004 / 12 / 20.98$ & 30.9 & $0.6-0.9$ & 3 & $300 \mathrm{~s}$ & 1.13 \\
\hline
\end{tabular}

\section{Observations}

We obtained visible and near-infrared spectra of (3200) Phaethon with the $4.2 \mathrm{~m}$ William Herschell telecope (WHT), the $2.50 \mathrm{~m}$ Nordic Optical Telescope (NOT), and the $3.6 \mathrm{~m}$ Telescopio Nazionale Galileo (TNG), at "El Roque de los Muchachos" Observatory (Canary Islands, Spain). The observational circumstances are listed in Table 1. Phaethon was observed in the visible spectral range on two different nights using two different telescopes. It was first observed with the WHT telescope on 14.844 November 2003 UT, using the red arm of ISIS spectrograph, with a low resolution $\left(158\right.$ lines $\left.\mathrm{mm}^{-1}\right)$ grating covering the 0.52 to $0.95 \mu \mathrm{m}$ spectral range. A $3^{\prime \prime}$ width slit was used. The slit was oriented in the parallactic angle and the tracking was at the object proper motion. Three spectra of $300 \mathrm{~s}$ exp. time each, and shifting the object $5^{\prime \prime}$ in the slit direction between exposures to better correct the fringing, were obtained. Spectral data reduction was done using standard IRAF package procedures. Images were over-scan and bias corrected, and flat-field corrected using lamp flats. The twodimensional spectra were extracted, sky background subtracted, and collapsed to one dimension. The wavelength calibration was done using the neon and argon lamps. The three spectra of Phaethon, obtained at different positions of the slit, were compared to discard any possible problems like a background object, and finally averaged. The reflectance spectrum was obtained by dividing the spectrum of Phaethon by the spectrum of the solar analogue star 16 Sygnus B obtained the same night at a similar airmass. The reflectance spectrum (see Fig. 1) was normalized at $0.55 \mu \mathrm{m}$ and smoothed using a smoothing box-car of 5 pixels.

Visible spectra were also obtained with the NOT telescope on 20.97 December 2004 UT, using ALFOSC (Andalucia Faint Object Spectrograph and Camera) with a low resolution (300 lines $\mathrm{mm}^{-1}$ ) grism disperser covering the $0.32-0.91 \mu \mathrm{m}$ spectral region. A $1.3^{\prime \prime}$ width slit oriented in the parallactic angle was used, and the tracking was at the object's proper motion. Three spectra of $300 \mathrm{~s}$ exp. time each were obtained using a second order blocking filter $(0.475 \mu \mathrm{m})$ and shifting the object $5^{\prime \prime}$ in the slit direction to better correct the fringing. Another spectrum of $900 \mathrm{~s}$ exp. time was also obtained without the second order blocking filter. The last spectrum was used to cover the $0.35-0.60 \mu \mathrm{m}$ region, while those obtained with the filter were used to cover the $0.60-0.9 \mu \mathrm{m}$ region. The overlap region $(0.50-0.65 \mu \mathrm{m})$ between two spectra gave an excellent agreement and was used to combine them. Data reduction was done as for WHT data. Solar analogue star Hyades 64 was observed the same night at a similar airmass and used to obtain the reflectance spectrum presented in Fig. 1. Also the G2 star SA98-978 (Landolt 1992) was observed and used as a solar analogue with very similar results. The reflectance spectra obtained had a spectral resolution of $3.21 \AA /$ pixel and were re-binned using a binning factor of 5 and normalized to unity at $0.55 \mu \mathrm{m}$. Notice that in the overlapping region the spectra from the WHT and from the NOT are essentially identical.

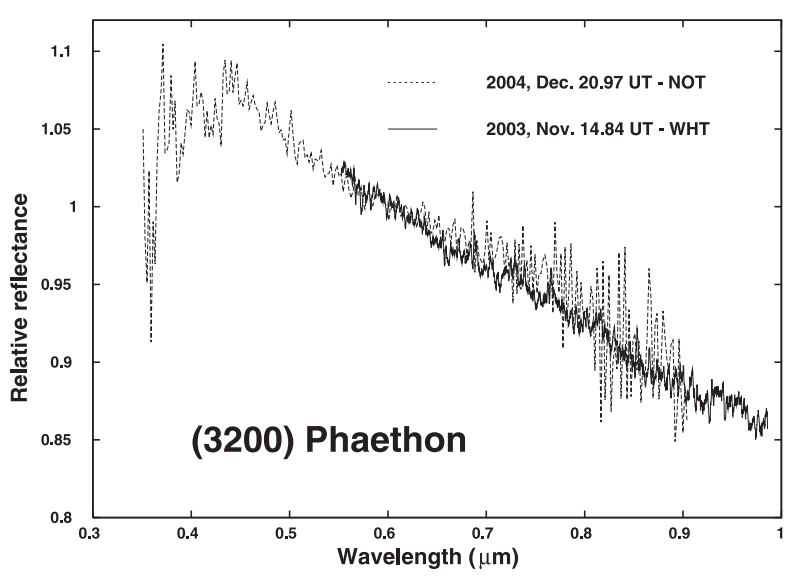

Fig. 1. Visible reflectance spectra obtained with the WHT and NOT telescopes on 14.844 November 2003 and 20.97 December 2004 UT respectively. Both spectra are essentially identical in the overlapping region.

The near-infrared low resolution spectrum was obtained on 11.917 December 2004 UT, using NICS, the Near-Infrared Camera and Spectrometer with its high throughput, low resolution spectroscopic mode and with an Amici prism disperser (Oliva 2001), which yields a complete 0.8-2.4 $\mu \mathrm{m}$ spectrum. The slit was oriented in the parallactic angle, and the tracking was at Phaethon's proper motion. A $1.5^{\prime \prime}$ slit width corresponding to a spectral resolving power $R \simeq 34$ quasi-constant along the spectrum was used. The observing and reduction procedures were as described in Licandro et al. (2002). The acquisition consisted of an image of $90 \mathrm{~s}$ exposure time in one position of the slit (position $A$ ) and then another $90 \mathrm{~s}$ image offsetting the telescope by $10^{\prime \prime}$ in the direction of the slit (position $B$ ). This process was repeated and $2 A B B A$ cycles were acquired. The total exposure time was $480 \mathrm{~s}$. To correct for telluric absorption and to obtain the relative reflectance, the G stars Landolt 93-101, Landolt 112-1333, and Landolt 115-271 (Landolt 1992) were observed during the same night at a similar airmass. These Landolt stars were also observed on previous nights together with the solar analogue star P330E (Colina \& Bohlin 1997) and present similar spectra in the infrared region, so we used them as solar analogues. By dividing the spectra of Landolt 93-101 and Landolt $112-1333$ by that of Landolt $115-271$ and then normalizing them to unity around $1.6 \mu \mathrm{m}$, we observed that the resulting spectra were very flat, the atmosphere was very stable during the observations, and the uncertainty in the slope was smaller than $0.5 \% / 1000 \AA$. Finally, the spectrum of Phaethon was divided by the spectra of each solar analogue star, and the results were normalized at $1.6 \mu \mathrm{m}$ and averaged, thus obtaining the final relative reflectance spectrum. Sub-pixel offsetting was applied when dividing the target and star spectra to correct for errors in the wavelength calibrations due to instrumental flexures. To analyze the complete visible and near-infrared (VNIR) spectra of our 


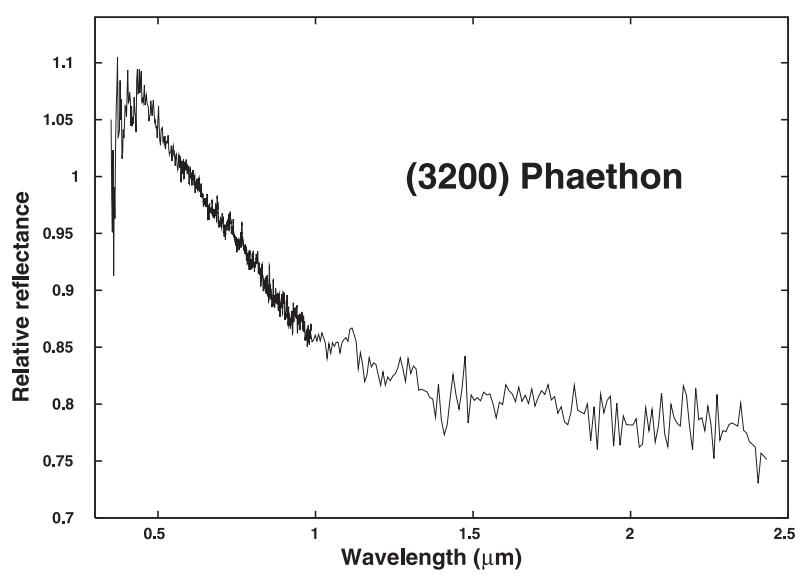

Fig. 2. The combined visible and near-infrared reflectance spectrum of (3200) Phaethon normalized to unity at $0.55 \mu \mathrm{m}$.

objects we used the NOT spectrum in the $0.32-0.56 \mu \mathrm{m}$ region, the WHT spectrum in the 0.52 to $0.95 \mu \mathrm{m}$ spectral range, and we overlapped the visible and near-infrared using the common interval between 0.78 and $0.95 \mu \mathrm{m}$ by performing a least-squares minimum procedure. The resulting complete VNIR spectrum is shown in Fig. 2.

\section{Our spectrum of Phaethon and comparison with other published spectra}

The most relevant characteristics of Phaethon's spectrum shown in Fig. 2 are: 1) in the visible region it is featureless at wavelengths longer than $0.43 \mu \mathrm{m}$, bluish with a negative slope $\left(S^{\prime}=\right.$ $-4 \pm 1$ where $S^{\prime}$ is the spectral gradient computed as in Luu $\&$ Jewitt (1990) considering the $0.55-0.9 \mu \mathrm{m}$ region, in units of $\% / 1000 \AA$ ), and the reflectance decreases below $0.43 \mu \mathrm{m}$. This corresponds to a B-type asteorid in the Bus (1999) taxonomy; 2) in the near-infrared region $(0.9-2.4 \mu \mathrm{m})$ the spectrum is also featureless, with a slightly blue spectral gradient $\left(S^{\prime}=-0.4 \pm 0.5\right.$ considering the $1.0-2.2 \mu \mathrm{m}$ region $) ; 3$ ) the spectrum is curved in the whole $0.43-2.4 \mu \mathrm{m}$ spectral region.

Other visible and near-infrared spectra of Phaethon have been published by Cochran \& Barker (1984), Luu \& Jewitt (1990), Chamberlin et al. (1996), Lazzarin et al. (1996), Hicks et al. (1998), Dumas et al. (1998), and Binzel et al. $(2001,2004)$. Also a near-infrared spectrum, obtained 10 days before ours within the "MIT-UH-IRTF Joint Campaign for NEO Reconnaissance", is available at their web page (http://smass.mit.edu/minus.html). In the near-infrared region, the MIT-UH-IRTF spectrum is very similar to ours (see Fig. 3), with a slightly bluer but consistent spectral gradient $\left(S^{\prime}=-0.7\right)$. The spectrum in the $H$ - and $K$-bands presented in Dumas et al. (1998) is also consistent with ours.

However, there are significant differences among the spectra observed in the visible region. Figure 4 shows our spectrum and published spectra of Phaethon. In the case of the Luu \& Jewitt (1990) spectrum we only plotted some points as the dispersion of the data is larger than the others and it is different. While the spectrum of Lazzarin et al. (1996) is not included for clarity, it is similar to those by Binzel et al. $(2001,2004)$ and that presented in this paper, but with lower $\mathrm{S} / \mathrm{N}$. At wavelengths longer than $0.6 \mu \mathrm{m}$ the avaliable spectra are similar, $\left(S^{\prime}=-6.8,-1.7\right.$, and -4.0 for the spectra presented by Binzel et al. (2001, 2004), and this work, respectively) with a mean $S^{\prime}=-4.2 \pm 2.55$. Considering that an uncertainty of 2 in $S^{\prime}$ in the visible region is

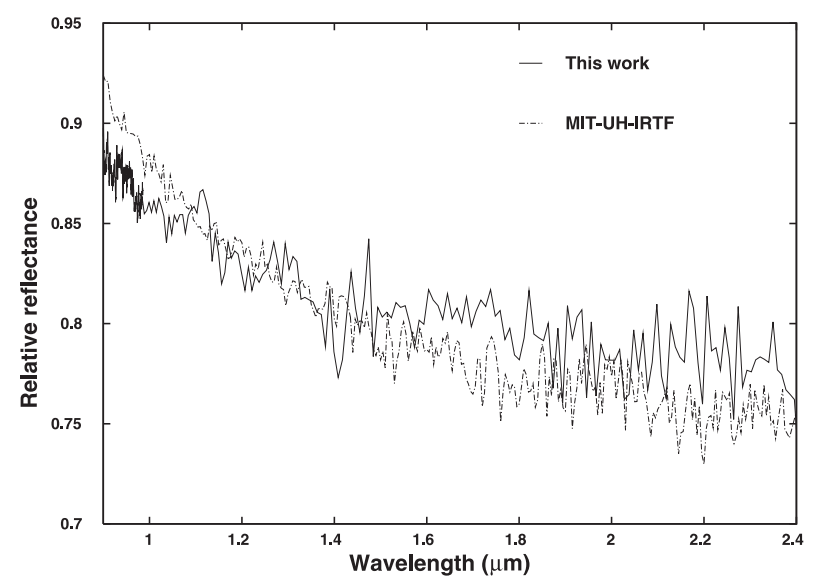

Fig. 3. Near-infrared reflectance spectrum of (3200) Phaethon presented in this paper, compared to Phaethon's spectrum obtained with the IRTF telescope by the "MIT-UH-IRTF Joint Campaign for NEO Reconnaissance". The MIT-UH-IRTF spectrum was renormalized to fit ours in the $1.2-1.4 \mu \mathrm{m}$ region. Both are very similar with a difference in slope that is consistent with the observational uncertainties.

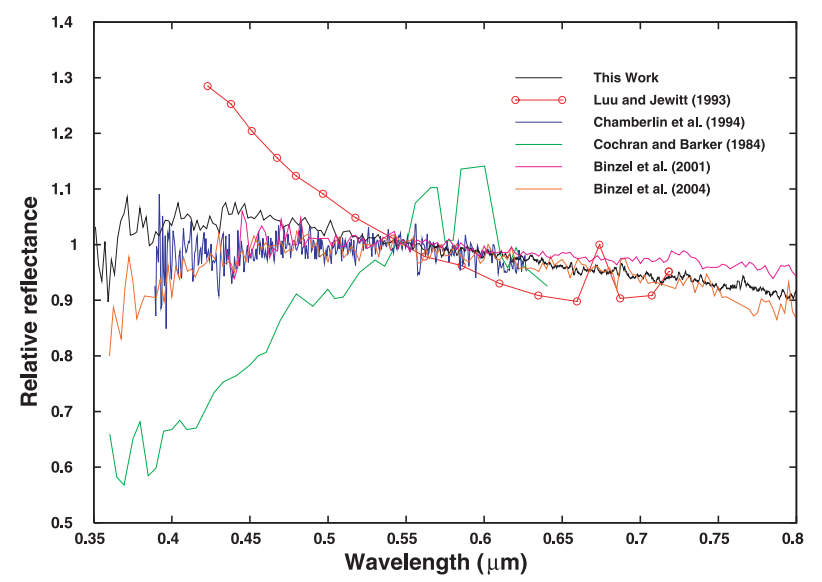

Fig. 4. Comparison of the visible reflectance spectrum of (3200) Phaethon presented in this paper with some of the already published spectra. Notice the differences in the UV region that suggest surface inhomogeneities (see text).

not unusual, we cannot claim a real slope variation longward of $0.6 \mu \mathrm{m}$. However, in the blue region the differences are greater. There are clear slope differences shortward of $0.55 \mu \mathrm{m}$. While the spectra from Chamberlin et al. (1996), Lazzarin et al. (1996), Binzel et al. (2001, 2004), and this work present a slightly red slope, that from Cochran \& Barker (1984) is very red $\left(S^{\prime}=23\right)$ and that from Luu \& Jewitt (1990) is very blue $\left(S^{\prime}=-11\right)$. Also considering the spectra from Chamberlin et al. (1996), Binzel et al. (2001), and this work, which look similar, we notice a difference in the position of the maximum. Chamberlin et al. (1996) and Binzel et al. (2001) are almost identical within the uncertainties, and present a maximum near $0.5 \mu \mathrm{m}$, while ours presents a maximum at about $0.45 \mu \mathrm{m}$ (see Fig. 4). Because these spectral variations are larger than the observational uncertainties, we consider them real; however, it would be desirable to confirm this spectral behavior (preferably with a homogeneous set of observations) and rule out any unidentified systematic uncertainties. 


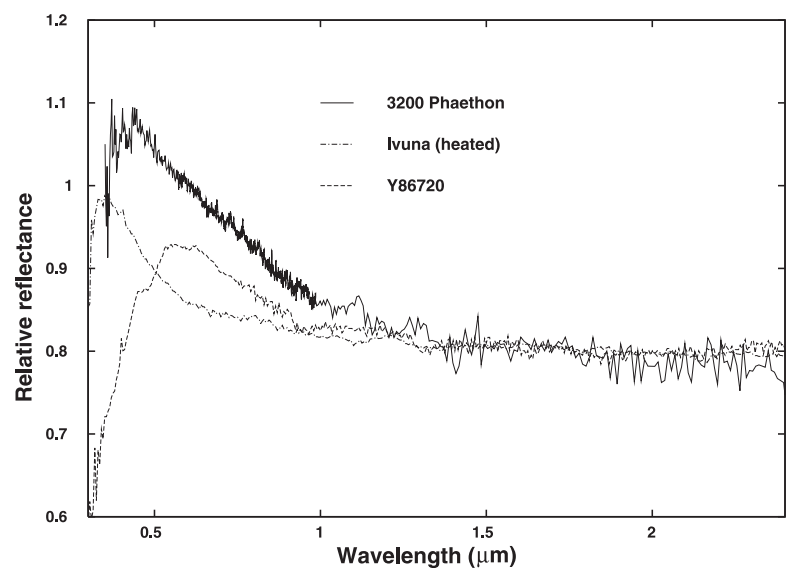

Fig. 5. Reflectance spectrum of (3200) Phaethon compared whith the spectra of two meteorite samples from the RELAB that present a similar shape, the CI meteor Ivuna heated to $700{ }^{\circ} \mathrm{C}$ and the unusual $\mathrm{CI} / \mathrm{CM}$ meteor Yamato-86720. The reflectance spectra of the meteorite samples were normalized to fit Phaethon's in the infrared for comparison purposes.

\section{Comparison with meteorites}

Aiming to infer the surface composition of Phaethon, we compared the reflectance spectrum presented in this paper with the meteorite spectra in the RELAB public database (Pieters \& Hiroi 2004), which contains 802 meteorite samples. A first order automatic search was performed by interpolating both the meteorite and the asteroid spectra to a common wavelength sampling in the spectral range covered by the asteroid spectrum. The dispersion between the resampled asteroid and meteorite spectra were then calculated for each spectrum in the database and sorted in ascending order of dispersion. A visual inspection of the plots of the asteroid and meteorites according to the resulting list was then performed. Although, no close spectral match was found among the meteorites, we identified some meteoritic samples that present spectral similarities with Phaethon's spectrum, like all the Yamato-86720 samples, and Ivuna heated to $700{ }^{\circ} \mathrm{C}$ (see Fig. 5). These samples present an absorption in the UV and a slightly blue slope. The maxima of their spectra are not at the same wavelength of that of Phaethon's spectrum, but the general shape is similar. Yamato-86720 is an unusual CI/CM meteorite that likely has been heated up to $500-600{ }^{\circ} \mathrm{C}$ (Hiroi et al. 1996), while Ivuna is a CI meteorite (Hiroi et al. 2004). CI and CM carbonaceous chondrites have been aquoeously altered and are rich in hydrated silicates.

Hiroi et al. (1996) suggested that C, G, B, and F asteroids may be heated inner portions of once larger bodies and that common CI/CM meteorites may have come from the lost outer portions, which escaped extensive late-stage heating events. In addition, space weathering effects could be responsible for the observed spectral differences between $\mathrm{CI} / \mathrm{CM}$ meteorites and $\mathrm{C}$-, B-, G-, and F-type asteroids (Moroz 2005), e.g. irradiation of complex hydrocarbon materials with charged particles leads to carbonization of their surface layers, producing a reduction of their VNIR spectral slopes (Moroz et al. 2004). It is interesting to note that the blackbody temperature of an object at Phethon's perihelion distance $(0.14 \mathrm{AU})$ is $750^{\circ} \mathrm{K}$, suggesting that thermal methamorphosis of surface material is likely.

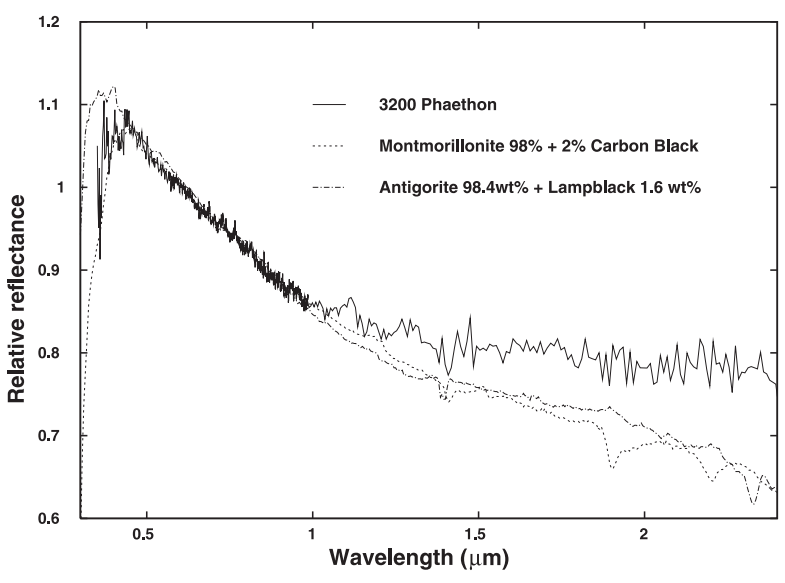

Fig. 6. Reflectance spectrum of (3200) Phaethon compared with the spectra of the two more similar man-made mineral mixtures found in the RELAB, the hydrated silicates Montmorillonite and Antigorite mixed with black carbon and lampblack (RELAB samples JB-CMP-023 and XT-TXH-056, respectively). Notice the similar shape of both mixtures, which peak at slightly different wavelengths, and Phaethon's spectrum. The reflectance spectra of the mixtures were normalized to fit Phaethon's in the visible for comparison purposes.

\section{Comparison with minerals and mineral mixtures}

We then decided to test if the spectrum of Phaethon has a good spectral match among the minerals and mixtures of minerals available. We used the same method described above, for all minerals, mixtures, and experimental samples available in RELAB, perfoming a total of 3090 spectral samples. Only two mixtures were found to resemble the spectrum of Phaethon, as shown in Fig. 6. Both are mixtures of hydrated silicates and carbon, the first containing 98\% Montmorillonite+2\% Carbon Black (RELAB sample JB-CMP-023), and the second one containing 98.4wt\% Antigorite + 1.6wt\% Lampblack (RELAB sample XT-TXH-056).

Montmorillonite is a soft phyllosilicate that typically forms in microscopic crystals, forming a clay. Its general chemical formula can be expressed as $(\mathrm{Na}, \mathrm{Ca})_{x}(\mathrm{Al}, \mathrm{Mg})_{2}\left(\mathrm{Si}_{4} \mathrm{O}_{10}\right)(\mathrm{OH})_{2} . n \mathrm{H}_{2} \mathrm{O}$, and has an average density of $2.35 \mathrm{~g} / \mathrm{cm}^{3}$. The water content is variable, and when water is absorbed by the crystals they tend to swell to several times their original volume. The near-infrared spectrum of Montmorillonites is dominated by strong molecular water bands at 1.4 and $1.9 \mu \mathrm{m}$ that are attenuated and even suppressed by adding only small amounts of carbon in the mixture. A good discussion on the spectral properties of mixtures of Montmorillonite and dark Carbon grains can be found in Clark (1983). The visible-NIR part of the spectrum of the mixture Montmorillonite $+2 \%$ Carbon Black fits the maximum of the spectrum of Phaethon around $0.4 \mu \mathrm{m}$ well, as well as the strong UV fall before it, and the visible-near-infrared portion up to about $1.5 \mu \mathrm{m}$. From $1.5 \mu \mathrm{m}$ to $2.5 \mu \mathrm{m}$, the spectrum of the mixture is slightly bluer than the spectrum of Phaethon.

The Antigorite is also a hydrated silicate, a monoclinic mineral from the kaolinite-serpentine group (chemical formula $\left.\left(\mathrm{Mg}, \mathrm{Fe}^{2+}\right)_{3} \mathrm{Si}_{2} \mathrm{O}_{5}(\mathrm{OH})_{4}\right)$, with average density $2.54 \mathrm{~g} / \mathrm{cm}^{3}$. On Earth, it can be found in many metamorphic and weathered igneous rocks. The reflectance spectrum of Antigorite alone also shows the 1.4 and $1.9 \mu \mathrm{m}$ water bands, but less intense than in Montmorillonites. Although the two maxima do not coincide (Fig. 6), the mixture 98.4wt\% Antigorite $+1.6 \mathrm{wt} \%$ Lampblack also reproduces the curvature and slope of the spectrum of 
Table 2. Surface composition given by the spectral modelling (see text). $p_{\mathrm{V}}$ is the visble albedo given by the model; Mont. is the fraction of Montmorillonite and black carbon mixture (RELAB sample JB-CMP-023); Ant. is the fraction of Antigorite and lampblack mixture (RELAB sample XT-TXH-056); Carbon is the fraction of black carbon; Graphite is the fraction of graphite; and Size is the diameter of the particles of each material used.

\begin{tabular}{lccccccccc}
\hline \hline Model & $p_{\mathrm{V}}$ & $\begin{array}{c}\text { Mont. } \\
\%\end{array}$ & $\begin{array}{c}\text { size } \\
(\mu \mathrm{m})\end{array}$ & $\begin{array}{c}\text { Ant. } \\
\%\end{array}$ & $\begin{array}{c}\text { size } \\
(\mu \mathrm{m})\end{array}$ & $\begin{array}{c}\text { Carbon } \\
\%\end{array}$ & $\begin{array}{c}\text { size } \\
(\mu \mathrm{m})\end{array}$ & $\begin{array}{c}\text { Graphite } \\
\%\end{array}$ & $\begin{array}{c}\text { Size } \\
(\mu \mathrm{m})\end{array}$ \\
\hline model 1 & 0.09 & 83 & 75 & & & 17 & 100 & & \\
model 2 & 0.13 & & & 90 & 75 & 10 & 100 & & \\
model 3 & 0.10 & 80 & 75 & 5 & 75 & 7 & 100 & 8 & 75 \\
\hline
\end{tabular}

Phaethon up to about $1.5 \mu \mathrm{m}$ quite well. It is interesting to note that the density of Geminid meteors, which were produced by Phaethon, is considerably higher than that of any other meteor shower $\left(3.0 \pm 0.9 \mathrm{~g} / \mathrm{cm}^{3}\right.$, Bellot-Rubio et al. 2002), and similar to that of hydrated silicates.

\subsection{Models}

We developed spectral models to pursue the similarities with hydrated mineral mixtures discussed in the previous section. Modeling of the surface spectra of atmosphereless solar system objects to infer their composition can be a powerful analytical tool and has been discussed in a number of publications (e.g., Hapke 1981; Shkuratov et al. 1999, Cruikshank et al. 1998; Emery \& Brown 2004; Clark et al. 2004). We used the scattering theory described by Shkuratov et al. (1999) for areal mixtures. As in the comparison with the spectra of mineral mixtures, the main constraints are the overall shape of the spectrum and the albedo. Our models included four components (see Table 2): the two hydrated mineral mixtures discussed previously (Montmorillonite and Antigorite mixed with darkening components), amorphous carbon, and graphite. The optical constants for the amorphous carbon and the graphite are from Rouleu \& Martin (1991) and Draine (1985), respectively. Optical constants are not available for the two hydrated mineral mixtures, so to include them in our model, we inverted the Skhuratov model to obtain the imaginary index from the known albedo of the mixture assuming the real part of the refractive index is constant.

In Fig. 7, we plot three models with the compositions and particle sizes given in Table 2. The antigorite mix peaks at shorter wavelengths than the montmorillonite mix and is higher at longer wavelengths, suggesting a larger fraction of montmorillonite than antigorite. The amorphous carbon and graphite components help to lower the albedo and raise the spectrum at the longer wavelengths. Notice that, as suggested by Moroz et al. (2004), space weathering produces carbonization of the surface layers, thus carbon in the mixtures is reasonably expected. The best fit is obtained with a model that combines all four components.

Models yield geometric albedos $\left(p_{\mathrm{V}}=0.09-0.13\right.$, see Table 2) in agreement with Phaethon's albedo $\left(p_{\mathrm{v}}=0.098\right.$, Dumas et al. 1998; $p_{\mathrm{v}}=0.09-0.17$, Veeder et al. 1984). These models are not unique, they are not meant to strictly define the surface composition, but rather to be indicative of likely components and to allow comparisons with related objects that have been modeled in similar ways. Although we did not explore the complete parameter space, our comparisons with minerals in the previous section considerably narrowed the possible surface compositions and yielded very good approximations to the spectra. More complex mixtures may produce better fits, but our

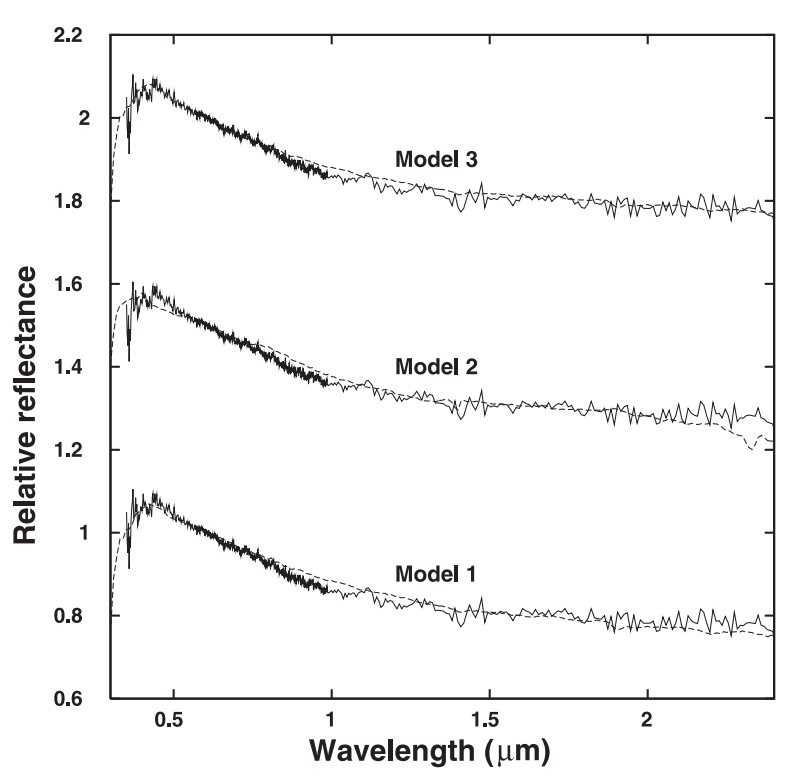

Fig. 7. Phaethon's spectrum with the three modeled spectra from Table 2 overplotted (dashed lines).

aim is simply to show that hydrated silicates can indeed produce reasonable fits.

We note that the hydrated silicates used in our model have strong absorptions in the $3 \mu \mathrm{m}$ region. It would be useful to attempt observations of 3200 Phaethon near $3 \mu \mathrm{m}$ near its aphelion, when it is sufficiently far from the Sun for that spectral range not to be dominated by thermal emission. Such observations would be more diagnostic not only of hydrated silicates, but also of any organic components. However, at aphelion, Phaethon is rather faint $(V \sim 20)$.

\section{Comparison with comets and activated asteroids}

To understand the cometary or asteroidal nature of Phaethon it is also important to compare its spectrum to that of comet nuclei and activated asteroids. Phaethon's spectrum shows important differences with the few comet nuclei properly observed at these wavelengths. In contrast with Phaethon, cometary nuclei have positive (red) slopes in the near-infrared (e.g., Campins et al. 2006, and references therein) and most have red slopes in the visible (Campins \& Fernandez 2002). Also neither of the two cometary nuclei with spectra shortward of $0.5 \mu \mathrm{m}$ (Abell et al. 2005; Campins et al. 2006) show an absorption toward the ultraviolet.

On the other hand, the spectrum of Phaethon is similar to those of the three other peculiar comet-asteroid transition objects: NEO 2001 YB5, an asteroid that has been associated with a meteor shower (Meng et al. 2004); NEO $4015 \mathrm{~W}-\mathrm{H}$, which showed cometary activity at the time of discovery, but 
has appeared asteroidal ever since (Fernandez et al. 1997); and 133P/Elst-Pizarro, a "main belt comet" (e.g., Hsieh \& Jewitt 2006). 2001 YB5 is a B-type NEA with a blue slope similar to that of Phaethon (Yang et al. 2003). The spectrum of $4015 \mathrm{~W}-\mathrm{H}$ in the visible spectral region $(0.35-0.7 \mu \mathrm{m})$ obtained by Chamberlin et al. (1996), is very similar to that of Phaethon in the same paper. There is no published spectrum of 133P/E-P, but published colors are compatible with B-type asteroids (Hsieh et al. 2003).

These differences with comets and similarities with other asteroids (albeit peculiar ones) argue in favor of Phaethon's active phase being more like an "activated asteroid" than a dying comet, akin to the behavior of the main belt comets in the Themis family (Hsieh \& Jewitt 2006). Interestingly, 36 members of the Themis family were spectroscopically studied in the $0.49-0.92 \mu \mathrm{m}$ range by Florczak et al. (1999). They found that the Themis asteroids are mostly B-, Cb-, and C-type, and about $50 \%$ of their sample showed evidence of aqueous alteration.

\section{Dynamical considerations}

The dynamical properties of Phaethon are compatible with the surface composition discussed above. Its Tisserand parameter respect to Jupiter, which is $T=4.18$, argues in favor of a main asteroid belt origin, rather than a cometary one. The boundary between cometary and asteroidal orbits is $T=3$; while most comets have $T<3$ (with a few exceptions that have $T$ sligthly larger than 3), most asteroids have $T>3$. It is difficult for Jupiter family comets to detach themselves from Jupiter and evolve to orbits with $T>3$. However, at least one active comet, 2P/Encke, has achieved such an orbit either due to the influence of nongravitational forces (Fernandez et al. 2002) or gravitational perturbations (Levison et al. 2006). According to the dynamical model by Bottke et al. (2002), the most likely source region for Phaethon is the $v 6$ resonance ( $52 \%$ probability), followed by the $3: 1$ resonance $(24 \%)$ and the Mars-crossing region (24\%) (see Weissman et al. 2002). Both the $v 6$ and the $3: 1$ resonances contain primitive asteroids with a significant fraction of hydrated members (e.g., Rivkin et al. 2002; Florczak et al. 1999).

\section{Conclusions}

In this paper we present visible and near-infrared spectra of asteroid (3200) Phaethon covering the $0.35-2.4 \mu \mathrm{m}$ range that provide new insights into the nature of this unusual object.

- a) Several arguments suggest that the surface of Phaethon contains hydrated minerals. As discussed in Sects. 4-6, there are close spectral similarities with hydrated mineral mixes, with hydrated meteorites, and with asteroids believed to have been aqueously altered. Model fits to the spectrum and albedo also indicate the presence of hydrated silicates. The density of the considered hydrated minerals is similar to that of the Geminid fireballs associated with Phaethon (which is significantly higher than the density of the meteors associated to other "normal" comets). The origin of these hydrated minerals in Phaethon may be similar to that of hydrated minerals in main belt asteroids, which are believed to result from aqueous alterations due to a heating event that melted existing ice (e.g., Rivkin et al. 2002). The small perihelion distance of Phaethon may be a heating mechanism that either produces or further processes the hydrated minerals.
- b) The dynamical properties of Phaethon are compatible with an asteroidal origin in regions of the main belt where asteroids with compatible surface composition are abundant. Both the $v 6$ and the 3:1 resonances contain primitive asteroids with a significant fraction of hydrated members.

- c) A possible spectral variability in the UV region is reported and discussed (see Sect. 3). If real, this variation is indicative of an inhomogeneous composition and/or physical properties (particle size, etc.) of the surface. Different hydrated silicates, with peaks ranging from about 0.4 to $0.6 \mu \mathrm{m}$ can produce such spectral variability (see Sect. 5). This surface inhomogeneous composition can be attributed to the hydration processes in the parent body of Phaethon and/or to further processing due to thermal heating. As Phaethon perihelion distance is small $(0.14 \mathrm{AU})$ heating by solar radiation could affect its surface composition (see Sect. 4, the meteorite samples found with similar spectra are artificially or naturally heated), and depending on Phaethon's pole orientation its surface can be inhomogeneously heated.

- d) Phaethon's spectrum shows important differences with the few comet nuclei properly observed at these wavelengths. The already obtained cometary nuclei spectra are redder and do not present an absorption toward the ultraviolet like Phaethon's spectrum does. On the other hand, the spectrum of Phaethon is similar to those of three other peculiar comet-asteroid transition objects: NEO 2001 YB5, NEO $4015 \mathrm{~W}-\mathrm{H}$, and 133P/Elst-Pizarro. These differences with comets and similarities with other asteroids (albeit peculiar ones) argue in favor of Phaethon's active phase being more like an "activated asteroid" than a dying comet.

The combination of dynamical and spectral connections to asteroids discussed in this paper seems compelling, and support the idea that Phaethon is an "activated" asteroid (like $4015 \mathrm{~W}-\mathrm{H}$ and the MBCs) scattered from the main-belt to the NEO population. The presence of hydrated silicates in its surface also supports that there was abundant liquid water in the past, and that its activity is driven by mechanisms similar to those proposed for MBCs (Hsieh \& Jewitt 2006). Some ice that remains in the interior of the asteroid may be triggered by impacts with small bodies that expose this ice to the Sun producing cometary-like activity.

Acknowledgements. We wish to thank Dr. Chamberlin for providing the ascii version of his Phaethon's spectra. H.C. acknowledges the support from grants from the National Science Founation and NASA's Planetary Astronomy program. This paper is based on observations made with the Italian Telescopio Nazionale Galileo (TNG) operated on the island of La Palma by the Centro Galileo Galilei of the CNAA (Consorzio Nazionale per l'Astronomia e l'Astrofisica) at the Spanish Observatorio del Roque de los Muchachos of the Instituto de Astrofisica de Canarias. We are grateful to all the technical staff and telescope operators for their assistance. T.M.-D. acknowledges the Conselho Nacional de Desenvolvimento Científico e Tecnológico/CNPq for the post-doc fellowship.

\section{References}

Abell, P., Fernandez, Y., Pravec, P., et al. 2005, Icarus, 179, 174 Bellot-Rubio, L., Martínez-González, M., Ruiz Herrera, L., et al. 2002, A\&A, 389,680

Binzel, R., Harris, A., Bus, S., \& Burbine, T. 2001, Icarus, 151, 139

Binzel, R., Rivkin, A., Stuart, J., et al. 2004, Icarus, 170, 259

Bottke, W. F., Morbidelli, A., Jedicke, R., et al. 2002, Icarus, 156, 399

Bowell, E., West, R., Heyer, H., et al. 1992, IAUC, 5585

Buie, M., \& Grundy, W. 2000, Icarus, 148, 324

Bus, S. J. 1999, Ph.D. Thesis, Massachusetts Institute of Technology

Campins, H., \& Swindle, T. 1998, Met. \& Plan. Sci., 33, 1201 
Campins, H., \& Fernandez, Y. 2002, EM\&P, 89, 117

Campins, H., Ziffer, J., Licandro, J., et al. 2006, AJ, 132, 1346

Chamberlin, A., McFadden, L., Schulz, R., Schleicher, D., \& Bus, S. 1995 , Icarus, 119, 173

Clark, R. 1983, J. Geophys. Res., 88, 10635

Clark, B., Bus, S., Rivkin, A., Shepard, M., \& Shah, S. 2004, AJ, 128, 3070

Cochran, A., \& Barker, E. 1984, Icarus, 60, 221

Colina, L., \& Bohlin, R. 1997, AJ, 113, 1138

Cruikshank, D., Roush, T., Bartholomew, M., et al. 1998 Icarus, 135, 389

Davies, J., Green, S., Meadows, A., Stewart, B., \& Aumann, H. 1984, Nature, 309,315

Draine, B. 1985, ApJS, 57, 587

Dumas, C., Owen, T., \& Barucci, A. 1998, Icarus, 133, 221

Emery, J., \& Brown, R. 2004, Icarus, 170, 131

Fernández, J., Gallardo, T., \& Brunini, A. 2002, Icarus, 159, 358

Fernández, Y., McFadden, L., Lisse, C., Helin, E., \& Chamberlin, A. 1997, Icarus, 128,114

Florczak, M., Lazzaro, D., Mothé-Diniz, T., Angeli, C., \& Betzler, A. 1999, A\&ASS, 134, 463

Green, S. 1983, IAUC, 3878

Gustafson, B. 1989, A\&A, 225, 533

Hapke, B. 1981, JGR, 86, 4571

Hicks, M., Fink, U., \& Grundy, W. 1998, Icarus, 133, 69

Hiroi, T., Zolensky, M., Pieters, C., \& Lipschutz, M. 1996, Meteor. Plan. Sci., 31,321

Hiroi, T., Pieters, C., Rutherford, M., et al. 2004, LPI, 35, 1616

Hsieh, H., \& Jewitt, D. 2005, ApJ, 624, 1093

Hsieh, H., \& Jewitt, D. 2006, Science, 312, 561

Hsieh, H., Jewitt, D., \& Fernandez, Y. 2006, AJ, 127, 2997
Hunt, J., Williams, I. P., \& Fox, K. 1985, MNRAS, 217, 533

Landolt, A. 1992, AJ, 104, 340

Lazzarin, M., Barucci, A., \& Doressoundiram, A. 1996, Icarus, 122, 122L

Levison, H., Terrell, D., Wiegert, P., Dones, L., \& Duncan, M. 2006, Icarus, 182, 161

Licandro, J., Ghinassi, F., \& Testi, L. 2002, A\&A, 388, L9

Luu, J., \& Jewitt, D. 1990, AJ, 99, 1985

Meng, H., Zhu, J., Gong, X., et al. 2004, Icarus, 169, 385

Moroz, L., Baratta, G., Strazzulla, G., et al. 2004, Icarus, 170, 214

Moroz, L. 2005, LPS, 36, 2056

Ohtsuka, K., Sekiguchi, T., Kinoshita, D., et al. 2006, A\&A, 450, 250

Oliva, E. 2001, Mem. SAIt., 71, 861

Pieters, C. 1983, J. Geophys Res., 88, 9534

Pieters, C., \& Hiroi, T. 2004, Lunar and Plan. Sci. Conf., 35, 1720

Rivkin, A., Howell, E., Vilas, F., \& Lebofsky, L. 2002, in Asteroids III, ed. W. Bottke, P. Paolicchi, R. Binzel, \& A. Cellino (U. of Arizona Press), 235

Rouleau, F., \& Martin, P. 1991, ApJ, 377, 526

Shkuratov, Y., Starukhina, L., Hoffmann, H., \& Arnold, G. 1999, Icarus, 137, 235

Thompson, W., Murray, B., Khare, B., \& Sagan, C. 1987, J. Geophys. Res., 92, 14933

Veeder, G., Kowal, C., \& Matson, D. 1984, LPI, 15, 409

Warren, S. G. 1986, Appl. Opt., 25, 2650

Weissman, P., Bottke, W., \& Levison, H. 2002, in Asteroids III, ed. W. F. Bottke Jr., A. Cellino, P. Paolicchi, \& R. P. Binzel (Tucson: University of Arizona Press), 669

Whipple, F. 1983, IAUC, 3881

Williams, I. P., \& Wu, Z. D. 1993, MNRAS, 264, 659

Yang, B., Zhu, J., Gao, J., et al. 2003, AJ, 126, 1086 